\title{
THE FUNNEL BOUNDARY OF MULTIVALUED DYNAMICAL SYSTEMS
}

\author{
P. E. KLOEDEN \\ Dedicated to Roman Matlak on the occasion of his 65th birthday
}

(Received 3 February; revised 23 June 1978)

Communicated by G. R. Morris

\begin{abstract}
Properties of the funnel boundary are investigated for multivalued dynamical systems defined axiomatically in terms of attainability set mappings on complete, locally compact metric state spaces. The set of regular boundary events is shown to be dense in the funnel boundary and theorems of Fukuhara and Zaremba on peripheral attainability are generalized to the systems considered here.
\end{abstract}

Subject classification (Amer. Math. Soc. (MOS) 1970): 34 C 35, 93 A 05.

\section{Introduction}

The topological properties of the set $F\left(x_{0}, t_{0}\right)$ of all state-time events $\left(x_{1}, t_{1}\right)$ that can be attained from an initial event $\left(x_{0}, t_{0}\right)$ by a multivalued dynamical system, called the funnel of the system through $\left(x_{0}, t_{0}\right)$, play a fundamental role in the qualitative theory of multivalued dynamical systems and have been extensively investigated for ordinary differential equations without uniqueness, ordinary differential control systems, contingent equations and stochastic differential equations. The properties of the funnel boundary $\partial F\left(x_{0}, t_{0}\right)$, notably the peripheral attainability of funnel boundary events, are of particular interest, especially in a control theoretic context in which the question of peripheral attainability is closely related to the existence of optimal controls with the bang-bang property.

The objective of this paper is to present a unifying account, with some new results, of the properties of the funnel boundary, in particular the peripheral 
attainability property, of abstract multivalued dynamical systems. The systems considered are defined axiomatically on complete, locally compact metric state spaces in terms of the properties of their attainability sets $A\left(x_{0}, t_{0}, t_{1}\right)$, the $t_{1}$-crosssections of the funnels $F\left(x_{0}, t_{0}\right)$. They include the general dynamical systems (GDS) of Barbashin (1948), the general control systems (GCS) of Roxin (1965) and the general semidynamical systems (GSDS) of Kloeden (1974a), which differ only in the successive weakening of assumptions on their backwards extendability in time. The axioms and basic properties of these systems are given in Section 2. In Section 3 the funnel $F\left(x_{0}, t_{0}\right)$ is defined in terms of the attainability sets $A\left(x_{0}, t_{0}, t_{1}\right)$ and the funnel boundary $\partial F\left(x_{0}, t_{0}\right)$ is decomposed into the set $R\left(x_{0}, t_{0}\right)$ of regular boundary events and the set $I\left(x_{0}, t_{0}\right)$ of irregular boundary events, where $\left(x_{1}, t_{1}\right) \in \partial F\left(x_{0}, t_{0}\right)$ is a regular boundary event if $x_{1} \in \partial A\left(x_{0}, t_{0}, t_{1}\right)$ and an irregular boundary event if not. Examples show that the set of irregular boundary events may be empty or nonempty. The main result of the section is Theorem 3.1 which says that the regular boundary events are dense in the funnel boundary.

In Section 4 a lemma required in the following section is proved for GDS for which all attainability sets are connected. Section 5 is concerned with the question of peripheral attainability and contains the main results of the paper. Theorem 5.1 is a generalization of a theorem of Fukuhara (1929) to GDS and shows that peripheral attainability, in fact a strong form of peripheral attainability, is implied by the connectedness of all attainability sets of the GDS. An example shows that this theorem does not hold for GSDS even though all nonempty attainability sets may be connected. Theorem 5.2 is the converse of Theorem 5.1. It generalizes to GDS a theorem of Zaremba (1935), which shows that the connectedness of all attainability sets is implied by the strong form of the peripheral attainability, but not, as examples verify, by the ordinary peripheral attainability property. Finally, Theorem 5.3 shows that no trajectory can lie in the set of irregular boundary events for a time interval of nonzero length. The last section considers the relationship between irregular boundary events and start events, that is, initial events from which the system cannot be extended backwards in time, of GSDS. The primary conclusion is that certain irregular boundary events, though not all, are caused by the presence of nearby start events exterior to the funnel and conversely that certain irregular boundary events imply the existence of nearby exterior start events.

\section{Multivalued dynamical systems}

The multivalued dynamical systems considered in this paper are defined axiomatically in terms of set valued attainability mappings $A: X \times \mathbf{R} \times \mathbf{R} \rightarrow X$ on a complete, locally compact metric state space $(X, d)$. An attainability set $A\left(x_{0}, t_{0}, t_{1}\right)$ denotes the set of all possible states which can be reached at time $t_{1}$ from an initial 
state $x_{0}$ at time $t_{0}$. Such dynamical systems have been investigated in many papers (for example, see Kloeden (1978) for a survey), with axioms based on the properties of the attainability sets of ordinary differential equations without uniqueness, ordinary differential control equations, contingent equations and stochastic differential equations. The axioms used differ slightly from paper to paper, but the following five are common to most:

Aхіом I. $A\left(x_{0}, t_{0}, t_{1}\right)$ is a nonempty, closed subset of $X$ for all $x_{0} \in X$ and $t_{0} \leqslant t_{1}$ in $\mathbf{R}$.

Axiom II. $A\left(x_{0}, t_{0}, t_{0}\right)=\left\{x_{0}\right\}$ for all $x_{0} \in X$ and $t_{0} \in \mathbf{R}$.

Axıом III. For all $x_{0} \in X$ and $t_{0} \leqslant t_{1} \leqslant t_{2}$ in $\mathbf{R}$

$$
A\left(x_{0}, t_{0}, t_{2}\right)=A\left(A\left(x_{0}, t_{0}, t_{1}\right), t_{1}, t_{2}\right)=\bigcup\left\{A\left(x_{1}, t_{1}, t_{2}\right) ; x_{1} \in A\left(x_{0}, t_{0}, t_{1}\right)\right\} .
$$

Axıом IV. $A$ is upper semicontinuous in $\left(x_{0}, t_{0}, t_{1}\right)$ with respect to the Hausdorff metric, that is given any $\varepsilon>0$ there exists $a \delta=\delta\left(x_{0}, t_{0}, t_{1}, \varepsilon\right)>0$ such that

$$
\rho^{*}\left(A\left(y_{0}, s_{0}, s_{1}\right), A\left(x_{0}, t_{0}, t_{1}\right)\right)<\varepsilon
$$

for all $y_{0} \in X$ and $s_{0} \leqslant s_{1}$ in $\mathbf{R}$ satisfying

$$
d\left(y_{0}, x_{0}\right)<\delta, \quad\left|s_{0}-t_{0}\right|<\delta, \quad\left|s_{1}-t_{1}\right|<\delta .
$$

Axıом V. $A$ is continuous in $t_{1}$ with respect to the Hausdorff metric for all $x_{0} \in X$ and $t_{0} \leqslant t_{1}$ in $\mathbf{R}$, that is given any $\varepsilon>0$ there exists $a \delta=\delta\left(x_{0}, t_{0}, t_{1}, \varepsilon\right)>0$ such that

$$
\rho\left(A\left(x_{0}, t_{0}, s_{1}\right), A\left(x_{0}, t_{0}, t_{1}\right)\right)<\varepsilon
$$

for all $t_{0} \leqslant s_{1}$ in $\mathbf{R}$ satisfying $\left|s_{1}-t_{1}\right|<\delta$.

In Axioms IV and V the Hausdorff metric $\rho$ on nonempty closed subsets of $X$ is defined by

$$
\rho(A, B)=\max \left\{\rho^{*}(A, B), \rho^{*}(B, A)\right\},
$$

where

$$
\rho^{*}(A, B)=\sup \{\rho(a, B) ; a \in A\}
$$

and

$$
\rho(a, B)=\inf \{d(a, b) ; \mathscr{C}, \in B\} .
$$

In Axiom I the attainability sets are assumed to be closed sets. In fact, as a consequence of the local compactness of $X$ and Axioms I, II and V they are compact sets. See Roxin (1965), Theorem 4.1. 
Multivalued dynamical systems satisfying Axioms I-V were considered by Kloeden (1974a-c) and called general semidynamical systems (GSDS) in view of their being defined only for future times, which is a feature common to many stochastic systems. Those satisfying the additional backwards extendability axiom:

Axıм VI-. For all $x_{1} \in X$ and $t_{0} \leqslant t_{1}$ in $\mathbf{R}$ there exists and $x_{0} \in X$ for which $x_{1} \in A\left(x_{0}, t_{0}, t_{1}\right)$.

were investigated by Roxin (1965) and called general control systems (GCS) as they were based on control systems governed by ordinary differential equations. With this backwards extendability axiom the domain of the attainability mapping $A$ can be extended to all of $X \times \mathbf{R} \times \mathbf{R}$ by defining $A\left(x_{0}, t_{0}, t_{1}\right)$ for $t_{1}<t_{0}$ by

$$
x_{1} \in A\left(x_{0}, t_{0}, t_{1}\right) \text { if and only if } x_{0} \in A\left(x_{1}, t_{1}, t_{0}\right) .
$$

Roxin showed that such a backwards extension of $A$ for $t_{1}<t_{0}$ satisfies all of the above axioms, except possibly Axiom V. See Roxin (1965), Section 5. Hence such attainability sets are closed, but not necessarily compact.

A stronger backwards extendability axiom

Axıом VI*. $A(x, t, t)$ satisfies Axioms $I-V$ for $t_{1} \leqslant t_{0}$ as well as for $t_{1} \geqslant t_{0}$.

in addition to Axioms I-V were used by Barbashin (1948) and Szegö and Treccani (1969) to define general dynamical systems (GDS). The systems were based on ordinary differential equations without uniqueness and, in view of Kneser's theorem, their attainability sets were also assumed to be connected sets. However, as the following example shows, the connectedness of attainability sets is independent of the above axioms.

EXAMPLE 2.1. Let $A$ be the GDS on $X=\mathbf{R}^{+} \times \mathbf{R}^{+}$constructed by patching together the straight line segments in $X \times \mathbf{R}$

$$
(x(t), y(t), t)=\left\{\begin{array}{lll}
\left(t-t_{0}+x_{0}, y_{0}, t\right) & \text { for } x_{0}<0 \text { and } t<t_{0}-x_{0} \\
\left(-t+t_{0}+x_{0}, y_{0}, t\right) & \text { for } x_{0}>0 \text { and } t<t_{0}+x_{0} \\
\left(0, t-t_{0}+y_{0}, t\right) & \text { for } x_{0}=0 \text { and } t \geqslant t_{0}-y_{0} .
\end{array}\right.
$$

See Fig. 1. Then for, say, $\left(x_{0}, y_{0}\right)=(0,0)$ and $t_{0}=0$, the attainability sets $A((0,0), 0, t)=\{-t,+t\}$ for all $t>0$. See also Example 4.1 in Roxin (1965).

Connectedness of attainability sets will not be assumed as an additional axiom here, but some of its consequences will be investigated in Sections 4 and 5 . Note 
that the attainability sets of Zaremba's familles complètes de courbes satisfy the axioms of a GDS on the finite time interval for which they are defined. See Zaremba (1935).

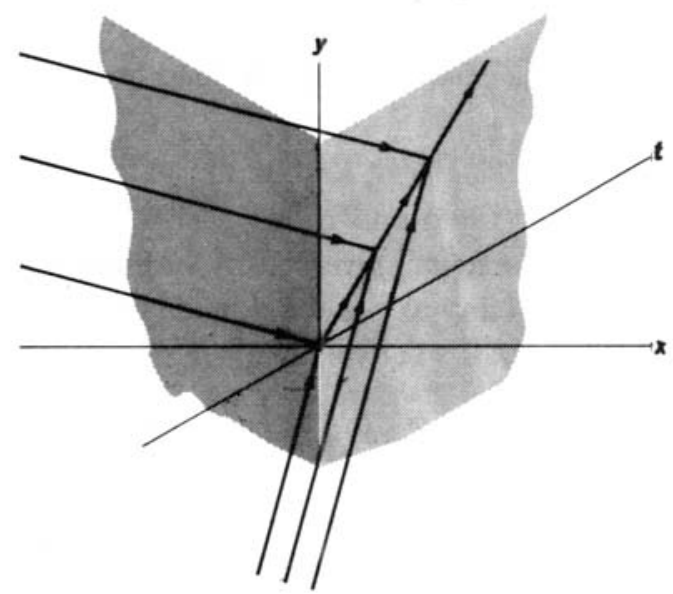

Fio. 1.

Historically, GDS were considered before GCS, which in turn were considered before GSDS. Roxin's justification for using a weaker backwards extendability axiom for GCS was that in a control context it is the future evolution of the system rather than its past evolution which is of primary interest. Even with the omission of any backwards extendability axiom for GSDS, attainability sets $A\left(x_{0}, t_{0}, t_{1}\right)$ can be defined for $t_{1}<t_{0}$ in the same way, namely (2.1), as for GCS, but for certain $x_{0}$ and $t_{0}$ they may be empty for all $t_{1}<t_{0}$. In this case $\left(x_{0}, t_{0}\right)$ is called a start event of the GSDS.

For differential systems attainability sets are usually defined in terms of the solutions or trajectories of the differential equations, whereas for multivalued dynamical systems trajectories are defined in terms of the attainability sets. Following Barbashin (1948) and Roxin (1965) a trajectory $\varphi$ of a multivalued dynamical system $A$ is defined as a single-valued mapping $\varphi:\left[t_{0}, t_{1}\right] \rightarrow X$ such that $\varphi(t) \in A(\varphi(s), s, t)$ for all $t_{0} \leqslant s \leqslant t \leqslant t_{1}$. Continuity of such trajectories follows from, Axioms II and IV (see Roxin (1965), Lemma 6) and their existence from the following theorem (see Barbashin (1948), Section 3, Theorem 1 for GDS; Roxin (1965) Theorem 6.1 for GCS and Kloeden (1974a), Theorem 4.1 for GSDS).

THEOREM 2.1. If $x_{1} \in A\left(x_{0}, t_{0}, t_{1}\right)$ for some $x_{0}, x_{1} \in X, t_{0}<t_{1}$ in $\mathbf{R}$ and multivalued dynamical system $A$, then there exists a trajectory $\varphi:\left[t_{0}, t_{1}\right] \rightarrow X$ of $A$ with $\varphi\left(t_{0}\right)=x_{0}$ and $\varphi\left(t_{1}\right)=x_{1}$. 
For GSDS all trajectories can be extended for all future times and for both GDS and GCS they can be extended for all future and all past times. Let $\Phi\left(x_{0}, t_{0}\right)$ denote the set of all such extended trajectories with $\varphi\left(x_{0}\right)=x_{0}$ for a given multivalued dynamical system. By the following theorem of Barbashin this set is sequentially compact (see Barbashin (1948), Theorem 2 for GDS; Roxin (1949), Theorem 6.2 for GCS and Kloeden (1974a), Theorem 4.2 for GSDS).

THEOREM 2.2. Let $K$ be a nonempty compact subset of $X$, let

$$
\left\{\varphi_{n}\right\} \subset \Phi\left(K, t_{0}\right)=\bigcup\left\{\Phi\left(x_{0}, t_{0}\right) ; x_{0} \in K\right\}
$$

for some multivalued dynamical system $A$ and let $[a, b]$ be a compact interval (with $t_{0} \leqslant a$ if $A$ is not a GDS). Then there exists a subsequence $\left\{\varphi_{n}\right\}$ and a trajectory $\varphi_{0} \in \Phi\left(K, t_{0}\right)$ such that $\varphi_{n}(t) \rightarrow \varphi_{0}(t)$ as $j \rightarrow \infty$ uniformly in $a \leqslant t \leqslant b$.

\section{The funnel boundary}

In the sequel let $A$ denote a fixed, but otherwise arbitrary multivalued dynamical system, which satisfies at least Axioms I-V, and also the condition that

$$
\partial A\left(x_{0}, t_{0}, t_{1}\right) \neq \varnothing
$$

for all $\left(x_{0}, t_{0}, t_{1}\right)$ in the domain of definition of $A$.

For such a multivalued dynamical system $A$ and any event $\left(x_{0}, t_{0}\right)$ in the state-time space $X \times \mathbf{R}$, the subset $F\left(x_{0}, t_{0}\right)$ of $X \times \mathbf{R}$ defined by

$$
F\left(x_{0}, t_{0}\right)=\bigcup\left\{A\left(x_{0}, t_{0}, t_{1}\right) \times\left\{t_{1}\right\} ; t_{1} \in \mathbf{R}\right\}
$$

is called the funnel of $A$ through $\left(x_{0}, t_{0}\right)$. This name is due to Kamke (1932), Seite 66 for ordinary differential equations without uniqueness. An alternative name used sometimes is the zone of emission of $A$ from $\left(x_{0}, t_{0}\right)$. The funnel $F\left(x_{0}, t_{0}\right)$ through $\left(x_{0}, t_{0}\right)$ represents the totality of all state-time events attainable from $\left(x_{0}, t_{0}\right)$ in either time direction by the multivalued dynamical systems and, by Axioms I and V, is a nonempty, closed and connected subset of $X \times \mathbf{R}$.

The funnel boundary $\partial F\left(x_{0}, t_{0}\right)$ is the boundary in $X \times \mathbf{R}$ of the funnel $F\left(x_{0}, t_{0}\right)$ through $\left(x_{0}, t_{0}\right)$ and is a nonempty and closed, but not necessarily connected, subset of $X \times \mathbf{R}$. It plays an important role in multivalued dynamical systems, particularly those arising from a control situation in which it is desired to control the system from the initial event $\left(x_{0}, t_{0}\right)$ to a nonempty, closed target set $\Theta \subset X \times \mathbf{R}$ and to optimize a specified cost functional in doing so. In such situations the optimal terminal events lie in the (supposedly nonempty) intersection $\partial F\left(x_{0}, t_{0}\right) \cap \Theta$ and for sufficiently regular, nondegenerate control problems $\left(\varphi^{*}(t), t\right) \in \partial F\left(x_{0}, t_{0}\right)$ for all 
intermediate times $t$ where $\varphi^{*}$ is an optimal trajectory, a property called the principle of optimal evolution by Halkin (1964). See also Bushaw (1963) and Roxin (1963). A similar property holds for ordinary differential equations without uniqueness, for which Fukuhara (1929) has shown that all events on the funnel boundary are peripherally attainable, that is attainable by trajectories $\varphi$ for which $(\varphi(t), t)$ lies entirely on the funnel boundary for all intermediate times $t$. This same property also holds for contingent equations. See Davy (1972). For control systems governed by ordinary differential equations this is closely related to the optimal controls satisfying the bang-bang property, that is taking values only in the boundary of the constraint set, which gives them a particularly simple and easily implementable form.

For any $\left(x_{0}, t_{0}\right) \in X \times \mathbf{R}$ the funnel boundary $\partial F\left(x_{0}, t_{0}\right)$ can be decomposed into two disjoint components

$$
R\left(x_{0}, t_{0}\right)=\bigcup\left\{\partial A\left(x_{0}, t_{0}, t_{1}\right) \times\left\{t_{1}\right\} ; t_{1} \in \mathbf{R}\right\}
$$

and

$$
I\left(x_{0}, t_{0}\right)=\partial F\left(x_{0}, t_{0}\right) \backslash R\left(x_{0}, t_{0}\right),
$$

called, respectively, the sets of regular and irregular boundary events of $F\left(x_{0}, t_{0}\right)$ (Bushaw (1963) calls the latter conjugate events, a term not used here to avoid confusion with their unrelated namesakes in differential geometry). In view of (3.2) and (3.3) an equivalent definition for the set of irregular boundary events is

$$
I\left(x_{0}, t_{0}\right)=\mathrm{U}\left\{\operatorname{int} A\left(x_{0}, t_{0}, t_{1}\right) \times\left\{t_{1}\right\} ; t_{1} \in \mathbf{R}\right\} \cap \partial F\left(x_{0}, t_{0}\right) .
$$

By Axiom II the set $R\left(x_{0}, t_{0}\right)$ of regular boundary events is nonempty for all $\left(x_{0}, t_{0}\right) \in X \times \mathbf{R}$, whereas the set $I\left(x_{0}, t_{0}\right)$ of irregular boundary events may be empty or nonempty. The former occurs, for example, whenever $\Phi\left(x_{0}, t_{0}\right)$ consists of only one trajectory. The following example shows that the latter may occur.

EXample 3.1. Let $X=\mathbf{R}^{+}$have the relative topology from $\mathbf{R}$ and let $A$ be the GDS defined graphically in Fig. 2. Then $0 \in$ int $A(1,0,1)$ and $(0,1) \in \partial F(1,0)$, so $(0,1) \in I(1,0)$. A similar example where $X$ is a circle can be found in Bushaw (1963).

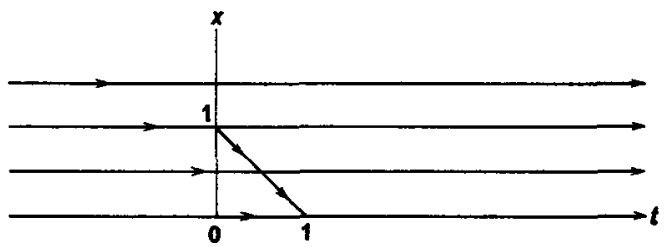

Frg. 2. 
The regular boundary events are, however, always dense in the funnel boundary. In the proof of this and elsewhere in the paper the product metric $D$ on $X \times \mathbf{R}$ defined by

$$
D\left(\left(x_{1}, t_{1}\right),\left(x_{2}, t_{2}\right)\right)=\max \left\{d\left(x_{1}, x_{2}\right),\left|t_{1}-t_{2}\right|\right\}
$$

will be used.

TheORem 3.1. $R\left(x_{0}, t_{0}\right)$ is dense in $\partial F\left(x_{0}, t_{0}\right)$ for all $\left(x_{0}, t_{0}\right) \in X \times \mathbf{R}$ and any multivalued dynamical system $A$, that is

$$
\overline{R\left(x_{0}, t_{0}\right)}=F\left(x_{0}, t_{0}\right)
$$

Proof. Suppose that $I\left(x_{0}, t_{0}\right)$ is nonempty, for otherwise there is nothing to prove, and let $\varepsilon>0$ and $\left(y_{0}, s_{0}\right) \in I\left(x_{0}, t_{0}\right)$.

For $s_{0}>t_{0}$, let $\delta=\delta\left(x_{0}, t_{0}, s_{0}, \varepsilon / 3\right)$ be the $\delta$ of Axiom $V$ corresponding to $\varepsilon / 3$. Without loss of generality it can be assumed to be less than both $\varepsilon / 3$ and $s_{0}-t_{0}$, so for $\left|s-s_{0}\right|<\delta$

$$
\rho\left(A\left(x_{0}, t_{0}, s\right), A\left(x_{0}, t_{0}, s_{0}\right)\right)<\varepsilon / 3 .
$$

Also as $\left(y_{0}, s_{0}\right) \in \partial F\left(x_{0}, t_{0}\right)$ there exists at least one event

$$
\left(y^{*}, s^{*}\right) \in S_{\epsilon / 3}\left(y_{0}, s_{0}\right) \cap \operatorname{ext} F\left(x_{0}, t_{0}\right),
$$

where $S_{\varepsilon / 3}\left(y_{0}, s_{0}\right)$ denotes the open sphere of radius $\varepsilon / 3$ about $\left(y_{0}, s_{0}\right)$ in $X \times \mathbf{R}$. Now $y^{*} \notin A\left(x_{0}, t_{0}, s^{*}\right)$ and since this set is compact there exists a $z^{*} \in \partial A\left(x_{0}, t_{0}, s^{*}\right)$ such that

$$
\begin{aligned}
d\left(y^{*}, z^{*}\right) & =\rho\left(y^{*}, A\left(x_{0}, t_{0}, s^{*}\right)\right) \\
& \leqslant \rho\left(y^{*}, A\left(x_{0}, t_{0}, s_{0}\right)\right)+\rho^{*}\left(A\left(x_{0}, t_{0}, s_{0}\right), A\left(x_{0}, t_{0}, s^{*}\right)\right) \\
& \leqslant d\left(y^{*}, y_{0}\right)+\rho^{*}\left(A\left(x_{0}, t_{0}, s_{0}\right), A\left(x_{0}, t_{0}, s^{*}\right)\right) \\
& <2 \varepsilon / 3
\end{aligned}
$$

in view of (3.4) and (3.5). Hence $\left(z^{*}, s^{*}\right) \in R\left(x_{0}, t_{0}\right)$ and

$$
\begin{aligned}
D\left(\left(z^{*}, s^{*}\right),\left(y_{0}, s_{0}\right)\right) & \leqslant d\left(z^{*}, y^{*}\right)+D\left(\left(y^{*}, s^{*}\right),\left(y_{0}, s_{0}\right)\right) \\
& <\varepsilon .
\end{aligned}
$$

For $s_{0}<t_{0}$ an analogous proof is valid for GDS but not for GCS nor GSDS as the attainability sets $A\left(x_{0}, t_{0}, s\right)$ need not be continuous in $s$ and compact for $s<t_{0}$. For them the proof proceeds as follows. Let $\varepsilon>0$ be sufficiently small so that $B=\overline{S_{a / 2}\left(y_{0}\right)}$ is a compact neighbourhood of $y_{0}$ in $X$ and is contained in $A\left(x_{0}, t_{0}, s_{0}\right)$. 
This is possible since $X$ is locally compact and $y_{0}$ is an interior point of $A\left(x_{0}, t_{0}, s_{0}\right)$. Then by Axiom IV there is an $0<\eta<\varepsilon$ such that the compact sets

$$
B(s)=A\left(x_{0}, t_{0}, s\right) \cap B
$$

are nonempty for $\left|s-s_{0}\right|<\eta$. (Note for GSDS the sets $A\left(x_{0}, t_{0}, s\right)$ are nonempty for $s_{0}-\eta<s<s_{0}$ and $\eta$ sufficiently small since not all events in $B \times\left(s_{0}-\eta, s_{0}\right] \cap \partial F\left(x_{0}, t_{0}\right)$ can be start events, for if they were the continuity of the attainability sets $A\left(x_{1}, t_{1}, s\right)$ in $s>t_{1}$ for $\left(x_{1}, t_{1}\right) \in B \times\left(s_{0}-\eta, s_{0}\right] \cap \operatorname{ext} \partial F\left(x_{0}, t_{0}\right)$ would be violated.)

By definition of $\partial F\left(x_{0}, t_{0}\right)$ there exists at least one event

$$
\left(y^{*}, s^{*}\right) \in S_{\eta}\left(y_{0}, s_{0}\right) \cap \partial F\left(x_{0}, t_{0}\right) .
$$

Hence $y^{*} \in B \backslash B\left(s^{*}\right)$ and as $B\left(s^{*}\right)$ is compact there exists a

$$
z^{*} \in \partial A\left(x_{0}, t_{0}, s^{*}\right) \cap B \subset \partial B\left(s^{*}\right) \text {. }
$$

Now $\left(z^{*}, s^{*}\right) \in R\left(x_{0}, t_{0}\right)$ and a $z^{*} \in B^{*}, d\left(z^{*}, y_{0}\right) \leqslant \varepsilon / 2<\varepsilon$. Also $\left|s^{*} \rightarrow s_{0}\right|<\eta<\varepsilon$. Hence

$$
D\left(\left(z^{*}, s^{*}\right),\left(y_{0}, s_{0}\right)\right)<\varepsilon .
$$

The following corollary will be used in Section 5 . In it the set $\partial F\left(K, t_{0}\right)$ denotes the boundary of $F\left(K, t_{0}\right)$ in $X \times \mathbf{R}$, not the set $\bigcup\left\{\partial F\left(x_{0}, t_{0}\right) ; x_{0} \in K\right\}$.

COROLlary 3.1. If $K$ is a nonempty compact subset of $X$, then the set $R\left(K, t_{0}\right)$ of regular boundary events of $F\left(K, t_{0}\right)$ is dense in $\partial F\left(K, t_{0}\right)$.

ProOF. The proof is the same as for Theorem 3.1 using the fact that $A\left(K, t_{0}, t_{1}\right)$ is a closed subset of $X$, which is continuous in $t_{1}$ (and hence compact) whenever $A\left(x_{0}, t_{0}, t_{1}\right)$ is continuous in $t_{1}$ for all $x_{0} \in K$. This follows from Theorems 4.3 and 4.4 of Roxin (1965).

\section{A lemma on connected attainability sets}

The following lemma is valid for those GDS for which every attainability set is connected, as for example in those GDS governed by ordinary differential equations without uniqueness. It will be required in the next section. The proof is in part based on that of Lemma 3.1 of Zaremba (1932).

LEMMA 4.1. Let $A$ be $a$ GDS for which $A\left(x_{0}, t_{0}, t_{1}\right)$ is connected for every $\left(x_{0}, t_{0}, t_{1}\right) \in X \times \mathbf{R} \times \mathbf{R}$. If $K$ is a nonempty compactum (that is compact connected set) in $X$, then $A\left(K, t_{0}, t_{1}\right)$ is a nonempty compactum in $X$ for all $t_{0}$ and $t_{1}$ in $R$. 
Proof. $A\left(K, t_{0}, t_{1}\right)$ is nonempty because $K$ and the sets $A\left(x_{0}, t_{0}, t_{1}\right)$ for all $\left(x_{0}, t_{0}, t_{1}\right) \in X \times \mathbf{R} \times \mathbf{R}$ are nonempty. It is compact because $K$ and the sets $A\left(x_{0}, t_{0}, t_{1}\right)$ for all $\left(x_{0}, t_{0}, t_{1}\right) \in X \times \mathbf{R} \times \mathbf{R}$ are compact and because $A$ is upper semicontinuous in its first variable. See Roxin (1965), Theorem 4.2.

Suppose $A\left(K, t_{0}, t_{1}\right)$ is not connected. Then there exist nonempty, closed and disjoint subsets $A_{1}$ and $A_{2}$ in $X$ such that $A\left(K, t_{0}, t_{1}\right)=A_{1} \cup A_{2}$. Moreover since $A\left(K, t_{0}, t_{1}\right)$ is compact, so are the sets $A_{1}$ and $A_{2}$. Hence

$$
\gamma=\inf \left\{d\left(a_{1}, a_{2}\right) ; a_{1} \in A_{1}, a_{2} \in A_{2}\right\}>0 .
$$

Let $K_{i}=\left\{x_{0} \in K ; A\left(x_{0}, t_{0}, t_{1}\right) \subset A_{i}\right\}$ for $i=1$ and 2 . Then by connectedness of the sets $A\left(x_{0}, t_{0}, t_{1}\right)$ for all $\left(x_{0}, t_{0}, t_{1}\right)$, the sets $K_{1}$ and $K_{2}$ are nonempty and disjoint. Also $K=K_{1} \cup K_{2}$.

Now let $\left\{x_{i}\right\}$ be a sequence in $K_{1}$ converging to $x_{0}$ and suppose that $x_{0} \notin K_{1}$. Then $x_{0} \in K_{2}$ and so $A\left(x_{0}, t_{0}, t_{1}\right) \subset A_{2}$ and

$$
\begin{aligned}
\rho^{*}\left(A\left(x_{i}, t_{0}, t_{1}\right), A_{2}\right) & \leqslant \rho^{*}\left(A\left(x_{i}, t_{0}, t_{1}\right), A\left(x_{0}, t_{0}, t_{1}\right)\right)+\rho^{*}\left(A\left(x_{0}, t_{0}, t_{1}\right), A_{2}\right) \\
& =\rho^{*}\left(A\left(x_{i}, t_{0}, t_{1}\right), A\left(x_{0}, t_{0}, t_{1}\right)\right) \\
& <\gamma / 2
\end{aligned}
$$

for all $i$ sufficiently large since $A$ is upper semicontinuous in its first variable. This, however, contradicts the fact that

$$
\rho^{*}\left(A\left(x_{i}, t_{0}, t_{1}\right), A_{2}\right) \geqslant \rho^{*}\left(A_{1}, A_{2}\right) \geqslant \gamma
$$

for all $i$ since $A\left(x_{i}, t_{0}, t_{1}\right) \subset A_{1}$ for all $i$. Hence $x_{0} \in K_{1}$ and so $K_{1}$ is closed. Similarly $K_{2}$ is closed.

This shows that $K$ is the union of two nonempty, disjoint and closed subsets $K_{1}$ and $K_{2}$, which contradicts the assumption that $K$ is connected. Hence $A\left(K, t_{0}, t_{1}\right)$ is connected.

The above proof is generally not valid for GCS as their attainability sets need not be compact for $t_{1}<t_{0}$. Also the connectedness of attainability sets of GCS for $t_{0}<t_{1}$ does not imply their connectedness for $t_{1}<t_{0}$. See Example 2.1 .

\section{Peripheral attainability}

The peripheral attainability of funnel boundary events of multivalued dynamical systems was first established by Fukuhara (1929) for ordinary differential equations without uniqueness and later by Roxin (1963), Theorem 3.1 for ordinary differential 
control systems. In both cases the result depended on the connectedness of attainability sets. In fact a stronger form of peripheral attainability follows from the connectedness of attainability sets. This was shown by Kamke (1932), Satz 5 for ordinary differential equations without uniqueness, by Zaremba (1935), Théorème 5.4 for his familles complètes de courbes and by Davy (1972), Theorem 7.3 for contingent equations. The following theorem shows that it holds for any GDS for which all attainability sets are connected.

THEOREM 5.1. Let $A$ be $a$ GDS for which $A\left(x_{0}, t_{0}, t_{1}\right)$ is connected for all $\left(x_{0}, t_{0}, t_{1}\right) \in X \times \mathbf{R} \times \mathbf{R}$. If $K$ is a nonempty compactum in $X$ and if

$$
\left(x_{1}, t_{1}\right) \in R\left(K, t_{0}\right) \subset \partial F\left(k, t_{0}\right)
$$

with $t_{0}<t_{1}$ (respectively $\left.t_{1}<t_{0}\right)$, then there exists a trajectory $\varphi_{0} \in \Phi\left(K, t_{0}\right)$ with $\varphi_{0}\left(t_{1}\right)=x_{1}$ and $\left(\varphi_{0}(t), t\right) \in R\left(K, t_{0}\right)$ for all $t_{0} \leqslant t \leqslant t_{1}$ (respectively $\left.t_{1} \leqslant t \leqslant t_{0}\right)$.

Proof. Let $t_{0}<t_{1}$; the proof for $t_{1}<t_{0}$ is analogous. The first step is to show that there exists a trajectory $\varphi \in \Phi\left(\partial K, t_{0}\right)$ with $\varphi\left(t_{1}\right)=x_{1}$. Since there is nothing to prove if $K$ has empty interior, suppose that $K$ has nonempty interior and that no such trajectory exists. As $\left(x_{1}, t_{1}\right) \in R\left(K, t_{0}\right)$, then $x_{1} \in \partial A\left(K, t_{0}, t_{1}\right)$ and so there exists a nonempty compactum $L$ in $X$ with

$$
\operatorname{L\cap } A\left(K, t_{0}, t_{1}\right)=\left\{x_{1}\right\} \text { and } L \cap \operatorname{ext} A\left(K, t_{0}, t_{1}\right) \neq \varnothing .
$$

Hence $A\left(L, t_{1}, t_{0}\right) \cap K \neq \varnothing, A\left(L, t_{1}, t_{0}\right) \cap \operatorname{ext} K \neq \varnothing$ and $A\left(L, t_{1}, t_{0}\right) \cap \partial K=\varnothing$, that is, $A\left(L, t_{1}, t_{0}\right) \subset$ int $K \cup$ ext $K$. This, however, contradicts the connectedness of $A\left(L, t_{1}, t_{0}\right)$, which holds on account of Lemma 4.1. Hence there exists at least one trajectory $\varphi \in \Phi\left(\partial K, t_{0}\right)$ with $\varphi\left(t_{1}\right)=x_{1}$.

The next step is to construct a sequence of trajectories $\left\{\varphi_{k}\right\}$ in $\Phi\left(x_{1}, t_{1}\right)$ satisfying

$$
\varphi_{k}\left(s_{k, i}\right) \in \partial A\left(K, t_{0}, s_{k, i}\right)
$$

for $i=0,1, \ldots, k$ where $s_{k, i}=t+i\left(t_{1}-t_{0}\right) / k$ for $i=0,1, \ldots, k$ and $k=1,2, \ldots$ To do this, let $k=1,2, \ldots$ be fixed. Then $x_{1} \in \partial A\left(A\left(K, t_{0}, s_{k, k-1}\right), s_{k, k-1}, t_{1}\right)$ and by Lemma $4.1 A\left(K, t_{0}, s_{k, k-1}\right)$ is connected. Hence by the same argument as in the first step there exists a trajectory $\varphi_{k, k-1}$ with $\varphi_{k, k-1}\left(s_{k, k-1}\right) \dot{\epsilon} \partial A\left(K, t_{0}, s_{k, k-1}\right)$ and $\varphi_{k, k-1}\left(t_{1}\right)=x_{1}$. Repeating this successively for $i=k-2, \ldots, 2,1$ with

$$
\varphi_{k, i+1}\left(s_{k, i}\right) \in \partial A\left(A\left(K, t_{0}, s_{k, i}\right), s_{k, i}, s_{k, i+1}\right)
$$

there exists a trajectory $\varphi_{k, i}$ with

$$
\varphi_{k, i}\left(s_{k, i}\right) \in \partial A\left(K, t_{0}, s_{k, i}\right) \text { and } \varphi_{k, i}\left(s_{k, i+1}\right)=\varphi_{k, i+1}\left(s_{k, i+1}\right) .
$$


Then

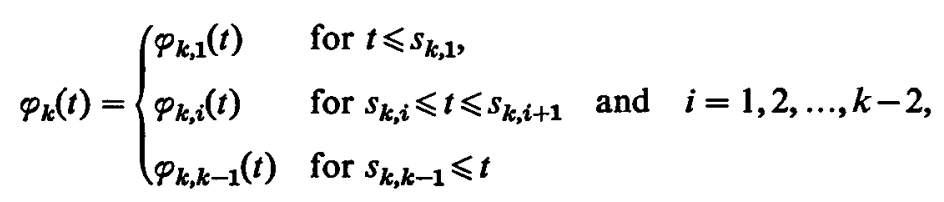

defines a trajectory $\varphi_{k} \in \Phi\left(x_{1}, t_{1}\right)$ which satisfies (5.1).

The final step is to use Theorem 2.2 on the sequence $\left\{\varphi_{k}\right\} \subset \Phi\left(x_{1}, t_{1}\right)$ to extract a convergent subsequence $\varphi_{k_{j}} \rightarrow \varphi_{0} \in \Phi\left(x_{1}, t_{1}\right)$. In view of (5.1), the continuity of $\varphi_{0}$ and the fact that the set $\left\{s_{k, i}: i=0,1, \ldots, k\right.$ and $\left.k=1,2, \ldots\right\}$ is dense in $\left[t_{0}, t_{1}\right]$, it follows that this trajectory $\varphi_{0}$ satisfies $\varphi_{0}(t) \in \partial A\left(K, t_{0}, t\right)$ for all $t_{0} \leqslant t \leqslant t_{1}$, that is, $\left(\varphi_{0}(t), t\right) \in R\left(K, t_{0}\right)$ for all $t_{0} \leqslant t \leqslant t_{1}$.

Under the same conditions of the above theorem, the irregular boundary events of $F\left(K, t_{0}\right)$ are also peripherally attainable, though not necessarily via irregular boundary events.

CoRollary 5.1. If $\left(x_{1}, t_{1}\right) \in I\left(K, t_{0}\right) \subset \partial F\left(K, t_{0}\right)$ with $t_{0}<t_{1}$ (respectively $t_{1}<t_{0}$ ), then there exists a trajectory $\varphi_{0} \in \Phi\left(\partial K, t_{0}\right)$ with $\varphi_{0}\left(t_{1}\right)=x_{1}$ and $\left(\varphi_{0}(t), t\right) \in \partial F\left(K, t_{0}\right)$ for all $t_{0} \leqslant t \leqslant t_{1}$ (respectively $\left.t_{1} \leqslant t \leqslant t_{0}\right)$.

PRoof. Let $t_{0}<t_{1}$; the proof for $t_{1}<t_{0}$ is similar. By Corollary 3.1 there exists a sequence $\left\{\left(z_{k}, s_{k}\right)\right\} \subset R\left(K, t_{0}\right)$ converging to $\left(x_{1}, t_{1}\right)$. Hence by Theorem 5.1 for each $k=1,2, \ldots$ there exists a trajectory $\varphi_{k} \in \Phi\left(\partial K, t_{0}\right)$ with $\varphi_{k}\left(s_{k}\right)=z_{k}$ and $\left(\varphi_{k}(t), t\right) \in R\left(K, t_{0}\right)$ for all $t_{0}<t \leqslant s_{k}$. Then by Theorem 2.2 there exists a subsequence $\left\{\varphi_{k}\right\}$ and a trajectory $\varphi_{0} \in \Phi\left(\partial K, t_{0}\right)$ such that $\varphi_{k}(t) \rightarrow \varphi_{0}(t)$ uniformly for $t_{0} \leqslant t \leqslant t_{1}$. For this trajectory $\varphi_{0}\left(t_{1}\right)=x_{1}$ and $\left(\varphi_{0}(t), t\right) \in \overline{R\left(K, t_{0}\right)}=\partial F\left(x_{0}, t_{0}\right)$ for all $t_{0} \leqslant t \leqslant t_{1}$.

There is no guarantee that $\left(\varphi_{0}(t), t\right)$ remains on the funnel boundary once $\left(x_{1}, t_{1}\right)$ has been reached. In Example 3.1 the single trajectory joining $(1,0)$ to $(0,1) \in \partial F(1,0)$ has $(\varphi(t), t) \in$ int $F(1,0)$ for all $t>1$. Another example of this involving an ordinary differential equation without uniqueness was constructed by Digel (1935).

Moreover without the connectedness of all attainability sets, the conclusion of Theorem 5.1 need not hold. In Example 2.1 the GDS has the peripheral attainability property, but not the stronger peripheral attainability property of Theorem 5.1 and in Example 5.2 of Zaremba (1932) the GDS which can be constructed from the complete family of curves does not have even the peripheral attainability property. In both examples there are attainability sets which are not connected. The following example shows that the conclusion of the theorem does not hold for GSDS even though all nonempty attainability sets are connected. 
EXAMPLe 5.1. Let $X=\mathbf{R}^{+}$have the relative topology in $\mathbf{R}$ and let $A$ be the GSDS defined graphically in Fig. 3, with $(0, t)$ a start event for all $t>2$. All nonempty attainability sets are connected, but none of the regular boundary events $(2-t, t)$ for $2<t<3$ in $R(1,0)$ are peripherally attainable.

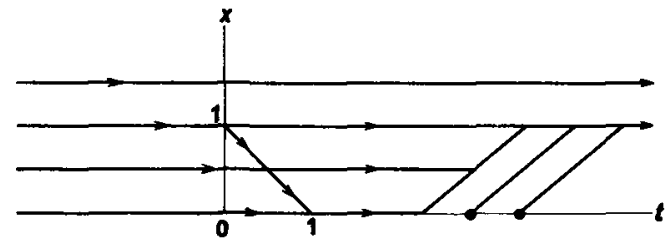

Fig. 3.

For GDS the converse of Theorem 5.1 is true. This was first shown by Zaremba (1935), Théorème 5.5 for his familles complètes de courbes.

THEOREM 5.2. Let $A$ be $a$ GDS such that for any nonempty compactum $K$ in $X$ and any $\left(x_{1}, t_{1}\right) \in R\left(K, t_{0}\right) \subset \partial F\left(K, t_{0}\right)$ with $t_{0}<t_{1}$ (respectively $\left.t_{1}<t_{0}\right)$ there exists a trajectory $\varphi_{0} \in \Phi\left(\partial K, t_{0}\right)$ with $\varphi_{0}\left(t_{1}\right)=x_{1}$ and $\left(\varphi_{0}(t), t\right) \in R\left(K, t_{0}\right)$ for all $t_{0} \leqslant t \leqslant t_{1}$ (respectively $\left.t_{1} \leqslant t \leqslant t_{0}\right)$. Then $A\left(x_{0}, t_{0}, t_{1}\right)$ is connected for all $\left(x_{0}, t_{0}, t_{1}\right) \in X \times \mathbf{R} \times \mathbf{R}$.

Proof. Suppose that $A\left(x_{0}, t_{0}, t_{1}\right)$ is not connected. Then there exist nonempty, compact and disjoint subsets $A_{1}$ and $A_{2}$ of $X$ such that $A\left(x_{0}, t_{0}, t_{1}\right)=A_{1} \cup A_{2}$. Hence by Condition (4.1) and the local compactness of $X$ there exists a nonempty compactum $B$ in $X$ such that $A_{1} \subset B, A_{2} \cap B=\varnothing$ and $A_{1} \cap \partial B=\varnothing$.

Let $t_{0}<t_{1}$; the case $t_{1}<t_{0}$ is handled similarly. As $A_{1} \subset B$ and $A_{1} \subset A\left(x_{0}, t_{0}, t_{1}\right)$, then $F\left(A_{1}, t_{1}\right) \subset F\left(B, t_{1}\right)$ and there exists a trajectory $\varphi_{1} \in \Phi\left(x_{0}, t_{0}\right)$ with $\varphi_{1}\left(t_{1}\right) \in A_{2}$ and $\left(\varphi_{1}(\tau), \tau\right) \in R\left(B, t_{1}\right)$ for some $t_{0}<\tau<t_{1}$. Hence there exists another trajectory $\varphi_{2} \in \Phi\left(\partial B, t_{1}\right)$ with $\varphi_{2}(\tau)=\varphi_{1}(\tau)$ and $\left(\varphi_{2}(t), t\right) \in R\left(B, t_{1}\right)$ for all $\tau \leqslant t \leqslant t_{1}$. Then

$$
\varphi_{0}(t)= \begin{cases}\varphi_{1}(t) & \text { for } t \leqslant \tau, \\ \varphi_{2}(t) & \text { for } \tau \leqslant t\end{cases}
$$

defines a trajectory $\varphi_{0} \in \Phi\left(\partial B, t_{1}\right) \cap \Phi\left(x_{0}, t_{0}\right)$. This, however, contradicts the fact that $A\left(x_{0}, t_{0}, t_{1}\right) \cap \partial B=\varnothing$. Hence $A\left(x_{0}, t_{0}, t_{1}\right)$ is connected for all

$$
\left(x_{0}, t_{0}, t_{1}\right) \in X \times \mathbf{R} \times \mathbf{R} \text {. }
$$

Example 2.1 shows that the conclusion of the above theorem need not hold if only peripheral attainability and not the strong form of peripheral attainability is assumed.

The final theorem for this section shows that the events $(\varphi(t), t)$ can never remain on the irregular part of the funnel boundary for a nonzero time interval for any 
trajectory and any multivalued dynamical system. Hence if as in Corollary 5.1 an irregular boundary event is peripherally attainable the corresponding trajectory arc must contain regular boundary events as well as irregular boundary events.

TheOREM 5.3. There exists no multivalued dynamical system $A$ with a trajectory $\varphi \in \Phi\left(x_{0}, t_{0}\right)$ such that $(\varphi(t), t) \in I\left(x_{0}, t_{0}\right)$ for $t_{1} \leqslant t \leqslant t_{2}$ for any $x_{0} \in X$ and $t_{0}, t_{1}<t_{2}$ in $\mathbf{R}$.

Proof. Suppose on the contrary that $A$ is a multivalued dynamical system with a trajectory $\varphi \in \Phi\left(x_{0}, t_{0}\right)$ such that $(\varphi(t), t) \in I\left(x_{0}, t_{0}\right)$ for $t_{1} \leqslant t \leqslant t_{2}$ for some $x_{0} \in X$ and $t_{0}, t_{1}<t_{2}$ in $\mathbf{R}$. Then for each $t_{1} \leqslant t \leqslant t_{2}$ there exists an $\varepsilon(t)>0$ such that

and

$$
S_{e}(\varphi(t)) \subset \operatorname{int} A\left(x_{0}, t_{0}, t\right)
$$

$$
S_{s}(\varphi(t), t) \cap \operatorname{ext} F\left(x_{0}, t_{0}\right) \neq \varnothing
$$

for all $0<\varepsilon<\varepsilon(t)$. Now the set $O_{1}=\bigcup\left\{S_{\varepsilon}(t)(\varphi(t), t) ; t_{1} \leqslant t \leqslant t_{2}\right\}$ is an open subset of $X \times \mathbf{R}$ containing the compact set $M_{1}=\bigcup\left\{(\varphi(t), t) ; t_{1} \leqslant t \leqslant t_{2}\right\}$. As $X$ is locally compact there thus exists (Kloeden (1974a), Lemma 5.3.1) an $\eta>0$ such that $M_{1} \subset S_{\eta}\left(M_{1}\right) \subset O_{1}$. Clearly $0<\eta<\varepsilon(t)$ for each $t_{1} \leqslant t \leqslant t_{2}$. Moreover $\eta$ can be chosen so $0<\eta<\left(t_{2}-t_{1}\right) / 5$. Then the open set

$$
\begin{aligned}
O_{2} & =\bigcup\left\{S_{\eta}(\varphi(t)) \times\{t\} ; t_{1}+\eta<t<t_{1}-\eta\right\} \\
& \subset \bigcup\left\{\operatorname{int} A\left(x_{0}, t_{0}, t\right) \times\{t\} ; t_{1}+\eta<t<t_{1}-\eta\right\} \\
& \subset F\left(x_{0}, t_{0}\right)
\end{aligned}
$$

contains the compact subset $M_{2}=\bigcup\left\{(\varphi(t), t) ; t_{1}+2 \eta \leqslant t \leqslant t_{2}-2 \eta\right\}$ of $M_{1}$ and so again by Lemma 5.3.1 of Kloeden (1974a) there exists a $0<\beta<\eta$ such that $M_{2} \subset S_{\beta}\left(M_{2}\right) \subset O_{2} \subset F\left(x_{0}, t_{0}\right)$. Hence for any $(\varphi(t), t) \in M_{2}$ and $0<\varepsilon<\beta$

$$
S_{s}(\varphi(t), t) \cap \operatorname{ext} F\left(x_{0}, t_{0}\right)=\varnothing
$$

contradicting (5.2) which holds since $M_{2} \subset M_{1} \subset I\left(x_{0}, t_{0}\right)$.

\section{Irregular boundary events and start events}

For GSDS the occurrence of some irregular boundary events is closely related to that of start events. In Example 5.1 the GSDS has start events in every $\varepsilon$-neighbourhood in $X \times \mathbf{R}$ of the irregular boundary event $(0,2)$ of the funnel $F(1,0)$. Moreover these start events all belong to $\operatorname{ext} F(1,0)$. This motivates the following theorem. 
THEOREM 6.1. Let $A$ be a GSDS and let $\left\{\left(y_{k}, s_{k}\right)\right\} \subset \operatorname{ext} F\left(x_{0}, t_{0}\right)$ be a sequence of start events converging to $\left(y_{0}, s_{0}\right)$, where $y_{0} \in \operatorname{int} A\left(x_{0}, t_{0}, s_{0}\right)$. Then $\left(y_{0}, s_{0}\right) \in I\left(x_{0}, t_{0}\right)$.

ProOF. As $\left(y_{k}, s_{k}\right) \rightarrow\left(y_{0}, s_{0}\right)$, for every $\varepsilon>0$ there exists a $k(\varepsilon)$ such that $\left(y_{k}, s_{k}\right) \in S_{\varepsilon}\left(y_{0}, s_{0}\right)$ for all $k \geqslant k(\varepsilon)$ and as $\left(y_{k}, s_{k}\right) \in \operatorname{ext} F\left(x_{0}, t_{0}\right)$ for all $k$, then

$$
S_{s}\left(y_{0}, s_{0}\right) \cap \operatorname{ext} F\left(x_{0}, t_{0}\right) \neq \varnothing \text {. }
$$

Hence $\left(y_{0}, s_{0}\right) \in \partial F\left(x_{0}, t_{0}\right)$ and as $y_{0} \in \operatorname{int} A\left(x_{0}, t_{0}, s_{0}\right)$, then $(y, s)\left(y_{0}, s_{0}\right) \in I\left(x_{0}, t_{0}\right)$.

The next example shows that the conclusion of the above theorem need not hold if the assumption that the start events belong to ext $F\left(x_{0}, t_{0}\right)$ is omitted.

EXAMPLE 6.1. Let $A$ be the GSDS on $X=\mathbf{R}$ defined graphically in Fig. 4, with $(0, t)$ start events for $0<t \leqslant 1$. Then $(0, t) \rightarrow(0,0) \in F(0,2)$ as $t \rightarrow 0+$ and $0 \in \operatorname{int} A(0,2,0)$, but $(0,0) \notin I(0,2)$. Indeed all of the start events and their limit events are contained in int $F\left(x_{0}, t_{0}\right)$.

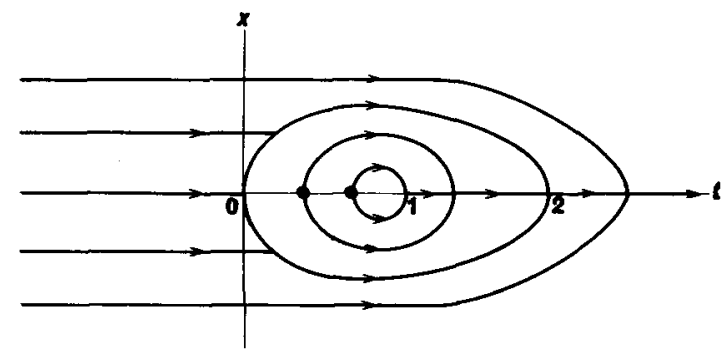

FIo. 4.

The converse of Theorem 6.1 does not hold, as can be seen from Example 3.1 of a GDS with an irregular boundary event and no start events. A partial converse does however hold. For this let

$$
I\left(x_{0}, t_{0}\right)=I^{+}\left(x_{0}, t_{0}\right) \cup I^{-}\left(x_{0}, t_{0}\right),
$$

where $\left(y_{0}, s_{0}\right) \in I^{+}\left(x_{0}, t_{0}\right)$ if

$$
S_{s}\left(y_{0}, s_{0}\right) \cap \operatorname{ext} F\left(x_{0}, t_{0}\right) \cap X \times\left[s_{0}, s_{0}+\varepsilon\right) \neq \varnothing
$$

and $\left(y_{0}, s_{0}\right) \in I^{-}\left(x_{0}, t_{0}\right)$ if

for every $\varepsilon>0$.

$$
S_{8}\left(y_{0}, s_{0}\right) \cap \operatorname{ext} F\left(x_{0}, t_{0}\right) \cap X \times\left(s_{0}-\varepsilon, s\right] \neq \varnothing
$$

In Example 5.1, $(0,1) \in I^{-}(1,0)$ and $(0,2) \in I^{+}(1,0)$. Also for the GSDS obtained from Fig. 3 by deleting the segment $X \times(1,2)$, the event $(0,1) \in I^{+}(1,0) \cap I^{-}(1,0)$, so these two sets may have points in common. The following theorem is a partial converse of Theorem 6.1. 
THEOREM 6.2. Let $A$ be $a$ GSDS and let $\left(y_{0}, s_{0}\right) \in I^{+}\left(x_{0}, t_{0}\right)$. Then there exists $a$ sequence $\left\{\left(y_{k}, s_{k}\right)\right\} \subset \operatorname{ext} F\left(x_{0}, t_{0}\right)$ of start events converging to $\left(y_{0}, s_{0}\right)$.

Proor. Suppose for some $\varepsilon>0$ there exist no start events in (6.1) and that $S_{s}\left(y_{0}\right) \subset \operatorname{int} A\left(x_{0}, t_{0}, s_{0}\right)$. Then for any sequence $\left(z_{k}, \tau_{k}\right)$ in (6.1) which converges to $\left(y_{0}, s_{0}\right)$, the backwards attainability sets $A\left(z_{k}, \tau_{k}, s\right)$ are nonempty as long as they remain in $S_{s}\left(y_{0}\right)$ and $\left|s-s_{0}\right|<\varepsilon$. Now $A\left(y_{0}, s_{0}, s_{0}\right)=\left\{s_{0}\right\}$ is compact and so by Roxin (1965), Theorem 5.1

$$
\rho\left(A\left(z_{k}, \tau_{k}, s\right), A\left(y_{0}, s_{0}, s_{0}\right)\right)<\varepsilon
$$

for sufficiently large $k$ and $s_{0} \leqslant s \leqslant \tau_{k}$. Hence $A\left(z_{k}, \tau_{k}, s\right)$ is nonempty for sufficiently large $k$ and

$$
A\left(z_{k}, \tau_{k s}, s\right) \subset S_{s}\left(y_{0}\right) \subset \operatorname{int} F\left(x_{0}, t_{0}, s_{0}\right) \text {. }
$$

Thus by Axiom III and Definition (2.1) of the backwards attainability sets, $z_{k} \in A\left(x_{0}, t_{0}, s_{k}\right)$ and so $\left(z_{k}, \tau_{k}\right) \in F\left(x_{0}, t_{0}\right)$ for all sufficiently large $k$. This, however, contradicts the choice of $\left(z_{k}, \tau_{k}\right)$ belonging to (6.1).

COROLLARY 6.2. If $A$ is a GDS or GCS, then $I^{+}\left(x_{0}, t_{0}\right)$ is empty for all $\left(x_{0}, t_{0}\right) \in X \times \mathbf{R}$.

For the irregular boundary events in $I^{-}\left(x_{0}, t_{0}\right)$, Example 3.1 shows that the conclusion of Theorem 6.2 need not hold, though it is not difficult to construct examples in which it does hold. For the irregular boundary events in $I^{+}\left(x_{0}, t_{0}\right)$ there are nearby regular boundary events which are not peripherally attainable at all or at least not peripherally attainable via regular boundary events.

\section{References}

E. A. Barbashin (1948), 'On the theory of general dynamical systems', Uchen. Zap. Moskov. Gos. Univ. 135, Mat. II, 110-133 (in Russian).

D. Bushaw (1963), 'Dynamical polysystems and optimization', Contrib. Diff. Equations 2, 351-365.

J. L. Davy (1972), 'Properties of the solution set of a generalized differential equation', Bull. Austral. Math. Soc. 6, 379-398.

E. Digel (1935), 'Zu einem Beispiel von Nagumo und Fukuhara', Math. Z. 39, 157-160.

M. Fukuhara (1929), 'Sur les systèmes d'équations différentielles, II', Japan. J. Math. 6, 269-299.

H. Halkin (1964), 'Topological aspects of optimal control of dynamical polysystems', Contrib. Diff. Equations 3, 377-385.

M. E. Kamke (1932), 'Zur Theorie der Systeme gewoehnlicher Differentialgleichungen, II', Acta Math. 58, 57-85.

P. E. Kloeden (1974a), On general semi dynamical systems (Ph.D. thesis, University of Queensland). 
P. E. Kloeden (1974b), 'General control systems without backwards extension', in Differential games and control theory (Lecture Notes in Pure and Applied Mathematics 10, Marcel Dekker, New York).

P. E. Kloeden (1974c), 'Generalised Markovian control systems', J. Austral. Math. Soc. 18, 485-491.

P. E. Kloeden (1977), 'General control systems', Proc. Instruct. Conf. on Math. Control Theory, Australian Nat. Univ., Canberra, edited by W. A. Coppel (Springer-Verlag, Berlin).

E. O. Roxin (1963), 'A geometric interpretation of Pontryagin's Maximum Principle', Inter. Symp. on Nonlin. Diff. Equations and Nonlinear Mech., Puerto Rico, edited by J. P. La Salle and S. Lefschetz (Academic Press, New York).

E. O. Roxin (1965), 'Stability in general control systems', J. Diff. Equations 1, 115-150.

G. P. Szegö and G. Treccani (1969), Semigruppi di trasformazioni multivoche (Lecture Notes in Mathematics 101, Springer-Verlag, Berlin).

S. K. Zaremba (1935), 'Sur certaines familles de courbes en relation avec la théorie des équations différentielles', Ann. Soc. Polon. Math. 15, 83-100.

School of Mathematical and Physical Sciences

Murdoch University, Murdoch

Western Australia, 6153 